\title{
Non-Newtonian Behavior of an Insoluble Monolayer: Effects of Inertia
}

\author{
Juan M. Lopez, ${ }^{*, 1}$ Reza Miraghaie, $\nmid$ and Amir H. Hirsa ${ }^{, 2}$ \\ * Department of Mathematics, Arizona State University, Tempe, Arizona 85287-1804; and †Department of Mechanical, Aerospace and Nuclear \\ Engineering, Rensselaer Polytechnic Institute, Troy, New York 12180-3590
}

Received September 12, 2001; accepted December 20, 2001; published online February 21, 2002

\begin{abstract}
Interfacial velocity measurements were performed in an optical annular channel, consisting of stationary inner and outer cylinders, a floor rotating at a constant rate, and a flat free surface on which an insoluble monolayer was initially spread. Measurements for essentially inviscid monolayers and some viscous monolayers on water show good agreement with numerical predictions for a Newtonian interface (Boussinesq-Scriven surface model) coupled to a bulk flow described by the Navier-Stokes equations. Here, we consider in detail a viscous monolayer, namely hemicyanine, and find that above a certain concentration, the monolayer does not behave Newtonian at a Reynolds number of about 250 . We show that the discrepancies between the measurements and predicted Newtonian behavior are not due to compositional effects (i.e., nonuniform monolayer distribution), Reynolds number (i.e., inertia and/or secondary flows), or surface dilatational viscosity (which does not play any role in the parameter regime investigated). We show prima facie evidence that the observed shear thinning nature of the velocity profile is associated with a phase transition at $C \approx 0.9 \mathrm{mg} / \mathrm{m}^{2}$ at low Reynolds numbers. At large Reynolds numbers $(\operatorname{Re}=8500)$, hemicyanine is found to flow like a viscous Newtonian monolayer on the air/water interface, with viscosity dependent only on the local concentration. $\odot 2002$ Elsevier Science (USA)

Key Words: surface shear viscosity; Marangoni stress; shear thinning; deep-channel surface viscometer.
\end{abstract}

\section{INTRODUCTION}

The problem of monolayer motion, whether induced at the interface itself (via surface tension gradients or moving walls) or driven by a bulk flow, continues to attract much attention. Interest in this problem stems from long-standing technological issues (e.g., film drainage in foam applications, optical coatings (1)) to emerging technologies in biological systems (e.g., flow in open microchannels $(2,3)$ ). The fundamental challenge in the development of predictive models continues to be our incomplete understanding of the intrinsic interfacial properties of monolayer-covered interfaces, in the context of a mechanistic constitutive relation for the interface (4).

\footnotetext{
${ }^{1}$ To whom correspondence should be addressed. E-mail: lopez@math.la. asu.edu.

${ }^{2}$ E-mail: hirsaa@rpi.edu.
}

Recently, our group has performed a series of measurements on several insoluble monolayers over a range of concentrations (and corresponding phase behavior) $(5,6)$. We have found that some monolayers, considered "well behaved" in classical literature (7), exhibit zero surface-excess viscosity, independent of the flow inertia (i.e., Reynolds number, Re). For example, monolayers of vitamin $K_{1}$ were found to have surface shear viscosity, $\mu^{\mathrm{s}}$, less than the sensitivity of the (low Re) deep-channel surface viscometer utilized (less than $10^{-4} \mathrm{sP}$ ). Good agreement was illustrated between velocity measurements and numerical predictions using the Boussinesq-Scriven surface model (8), confirming the strictly elastic nature of that monolayer over a wide range of $\operatorname{Re}$ (order $10^{2}-10^{4}$ ) and surface concentration $0<C \leq 1.5 \mathrm{mg} / \mathrm{m}^{2}$ (with a corresponding range in surface pressure of up to 10 dynes $/ \mathrm{cm}$ ). However, we have had mixed success with predictions of viscous monolayers when using a Newtonian model for the interface. Measurements and computations of stearic acid monolayers have shown good agreement, verifying the Newtonian behavior of that monolayer over a wide range of concentrations $\left(0<C \leq 1.6 \mathrm{mg} / \mathrm{m}^{2}\right)$ with surface shear viscosity that is only a function of the thermodynamic state (6). As will be shown in this paper, measurements with another viscous monolayer that has a more complicated equation of state (and presumably phase behavior), namely hemicyanine, exhibits shear thinning and possibly other non-Newtonian behavior. Shear thinning behavior has also been seen in low Re experiments with other Langmuir monolayers (9).

Here we analyze surface velocity profiles of a hemicyanine monolayer on an air/water interface. The flow geometry in the present study consists of stationary inner and outer cylinders, of radii $r_{\mathrm{i}}$ and $r_{\mathrm{o}}$, respectively, and a floor rotating at a constant angular velocity, $\Omega$. This geometry is essentially the deepchannel viscometer, except that here we are not restricted to small annular gap-to-depth ratios. In the range of angular velocity utilized, from the Stokes flow limit to large inertia where the secondary flow dominates the dynamics, the flow remains steady and axisymmetric and the interface remains flat (5). We compare the measured velocity profiles with predictions from a computational model that fully accounts for the secondary flow in the bulk (i.e., solve the Navier-Stokes equations in the bulk) and couple the bulk flow to a Newtonian interfacial flow model derived from the Boussinesq-Scriven constitutive relation. The 
measured nonlinear equation-of-state and surface shear viscosity for hemicyanine is incorporated into the model, and the surface advection-diffusion equation for insoluble monolayer transport on the interface is also solved. Although we have shown that when the interface is completely covered by the monolayer, the radial surface velocity in these flows diminishes to zero and that the radial stress balance at the interface can be replaced by the no-slip condition in the radial direction $(10,6)$, we do not make this modeling simplification in this paper in order to assess any contributions due to compositional effects (i.e., nonuniform monolayer distribution). Even if the radial velocity is zero when the interface is completely covered, there are still surfactant concentration gradients (due to the balance between advection by the bulk flow at the interface and Marangoni elasticity) which may contribute to the azimuthal stress balance since $\mu^{\mathrm{s}}$ is a (nonlinear) function of $C$; these potential contributions were not accounted for in [6]. Here, we a postiori validate the use of this model.

Apart from axisymmetry and flatness of the interface, a particularly attractive feature of the annular geometry is that when the interface is completely covered and the radial surface velocity is zero, then the surface viscosities ( $\mu^{\mathrm{s}}$ and surface dilatational viscosity, $\left.\kappa^{\mathrm{s}}\right)$ do not contribute to the radial stress balance. Hence, one does not need to determine $\kappa^{\mathrm{s}}$ for this model, and this is fortunate since $\kappa^{\mathrm{s}}$ is a particularly difficult intrinsic interfacial property to measure (1). The surface shear viscosity still plays an important role, as it determines the azimuthal stress balance. We incorporate $\mu^{\mathrm{s}}$ as measured by the standard deep-channel surface viscometer technique (which assumes a Newtonian interface). So our predictions are for a hemicyanine monolayer under the complete experimental conditions, assuming Newtonian behavior. Any substantial (i.e., allowing for experimental uncertainty and measurement biases) departures in the comparison of (azimuthal) velocity profiles from experiments and the model would then indicate non-Newtonian behavior. This type of analysis of the experiments is common in assessing the nonNewtonian characteristics of interfacial flow (11).

The numerical solution to the Navier-Stokes equations for the bulk flow allows us to examine a wide range of surface strain rate. The surface strain rate is essentially the component of vorticity normal to the surface and is proportional to the rate of rotation of the bottom (measured nondimensionally by the Reynolds number). Although the coupling between the bulk flow and the monolayer has been analyzed for the annular geometry by (12) in their study of the deep-channel viscometer (13), no previous study has systematically examined non-Newtonian interfacial hydrodynamics with nonlinear flow (i.e., inertia).

\section{EXPERIMENTAL APPARATUS AND METHODS}

A transparent annular channel, shown schematically in Fig. 1, was used for measuring the velocity field. The floor of this instrument consists of an optical-quality window. The stationary cylinders as well as the rotating cylinder, used to contain the liquid, are made of cast acrylic. In the annular region, the contact

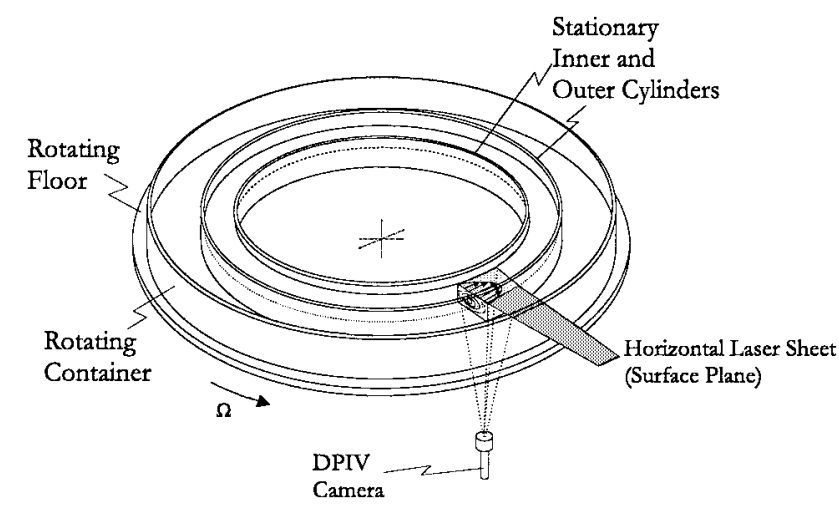

FIG. 1. Schematic of flow geometry.

line at both the inner and outer cylinders were fixed by machined groves and the interface was initially flat and found to deform less than $0.005 \pm 0.001 \mathrm{~cm}$ at the maximum floor rotation rate (5). The outer radius of the channel is $r_{\mathrm{o}}=9.82 \pm 0.01 \mathrm{~cm}$, the ratio of the radii is $r_{\mathrm{i}} / r_{\mathrm{o}}=0.776$, and the depth-to-gap ratio is $d /\left(r_{\mathrm{o}}-r_{\mathrm{i}}\right)=0.503$.

The flow on the air/water interface was measured using digital particle image velocimetry (DPIV) (14), by placing a thin laser light sheet $(<0.04 \mathrm{~cm})$ at the interface and imaging seeding particles from below (see Fig. 1). The seeding particles, 8.1- $\mu \mathrm{m}$ polystyrene (Duke Scientific, 7508A), were cleaned using a procedure detailed in (5).

Double-distilled water, at $22 \pm 1^{\circ} \mathrm{C}$, was used throughout the experiments. Tests of the purity of the water included measurements of the surface pressure, $\mathrm{pH}$, and resistivity and the results were similar to those reported in (5). A relatively stringent test of surface pressure of the (seeded) water sample was utilized. Specifically, the surface pressure of the water sample was measured following a rapid compression of the interface. A Langmuir trough, constructed of Teflon, was utilized and the surface tension was measured using an electrobalance (NIMA, PS4) and a Wilhelmy plate made of clean filter paper (NIMA). The water surface was compressed $11: 1$ in $55 \mathrm{~s}$ in the trough. The reduction in surface tension was found to be 0.1 dynes $/ \mathrm{cm}$, which is of the order of the noise of the instrument.

Monolayers of a hemicyanine dye were spread on the water and studied. The hemicyanine, of molecular weight $613.82 \mathrm{~g} /$ gmol (Sigma-Aldrich, 36683-8) was used as received, without further purification. The equation-of-state, $\sigma(C)$, measured in the Langmuir trough during a slow compression is presented in Fig. 2. For concentrations shown, the monolayer is well behaved and $\sigma(C)$ is the same during a (quasi-static) compression and subsequent expansion, to within an uncertainty of \pm 0.2 dynes/ $\mathrm{cm}$. The solid line in the figure corresponds to the curve fit

$$
\sigma(C)=\left\{\begin{array}{c}
65.9+6.4 \tanh (4.4(1-C / 0.77)) \text { dynes } / \mathrm{cm} \\
\text { for } C \leq 1.0 \mathrm{mg} / \mathrm{m}^{2} \\
60.3619-9.18661(C-1) \text { dynes } / \mathrm{cm} \\
\text { for } C>1.0 \mathrm{mg} / \mathrm{m}^{2}
\end{array}\right.
$$




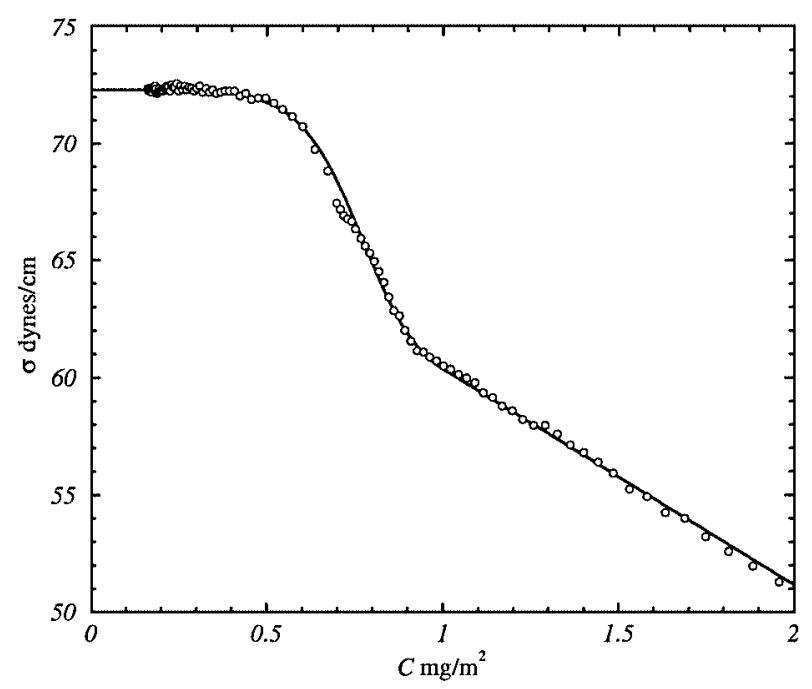

FIG. 2. Equation-of-state for a hemicyanine monolayer on an air/water interface at $22 \pm 1{ }^{\circ} \mathrm{C}$; circles are measurements (only one in five shown) using a Whilhelmy plate in a Langmuir trough and the solid line is the curve fit given by Eq. [1].

A pronounced phase transition is apparent at $C \approx 0.9 \mathrm{mg} / \mathrm{m}^{2}$. For convenience, this data is shown in Fig. 3 in terms of surface pressure $(\Pi=\sigma(0)-\sigma(C)$ dynes/cm) vs area per molecule ( $A, \AA^{2} /$ molecule).

The surface shear viscosity of hemicyanine monolayers was first examined using a conventional deep-channel surface viscometer $(13,12,15)$. A precision-machined, stainless steel channel, with $r_{\mathrm{o}}=5.074 \pm 0.001 \mathrm{~cm}, r_{\mathrm{i}} / r_{\mathrm{o}}=0.751$, and $d /\left(r_{\mathrm{o}}-\right.$ $\left.r_{\mathrm{i}}\right)=0.741$ was operated at $2 \pi / 60 \mathrm{rad} / \mathrm{s}(1 \mathrm{rpm})$, corresponding to a Reynolds number $\operatorname{Re} \approx 280$. The time for a single nonwetting particle (hollow ceramic microsphere, 3M, Z-Light W-1600,

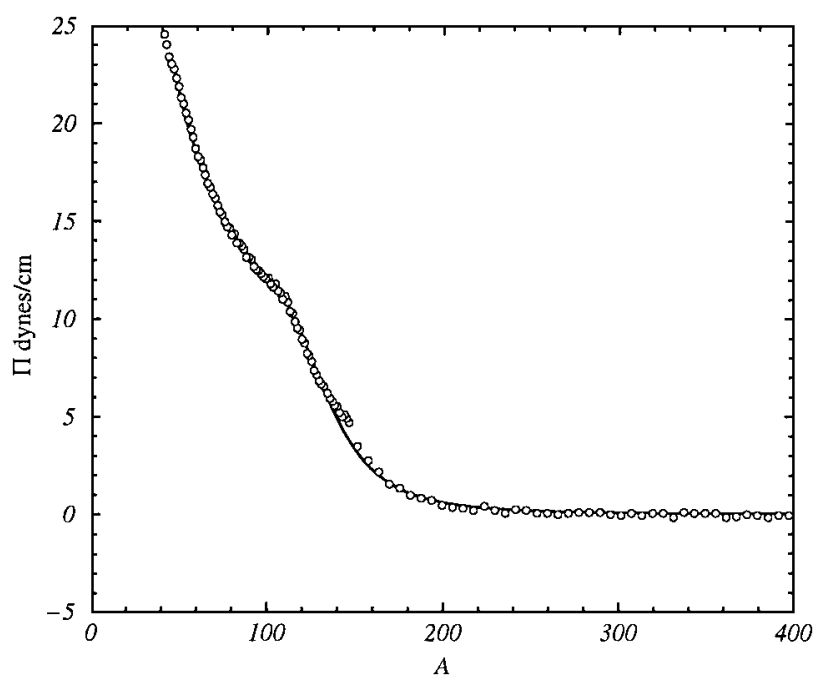

FIG. 3. Surface pressure vs area for a hemicyanine monolayer on an air/water interface at $22 \pm 1^{\circ} \mathrm{C}$; circles are measurements (only one in five shown) using a Whilhelmy plate in a Langmuir trough and the solid line corresponds to the curve fit given by Eq. [1].

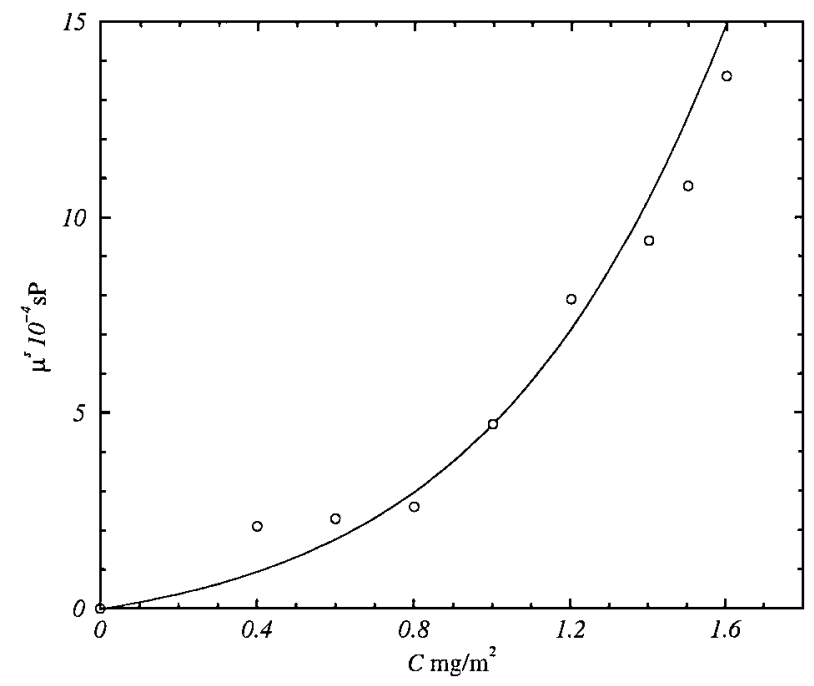

FIG. 4. Variation of surface shear viscosity, $\mu^{\mathrm{s}}$, with surface concentration of a hemicyanine monolayer on an air/water interface at $22 \pm 1{ }^{\circ} \mathrm{C}$; circles are measurements using a deep-channel viscometer and the solid line is the curve fit given by Eq. [2].

with a typical diameter of $200 \mu \mathrm{m}$ ) deposited at the center of the annulus was measured and the surface shear viscosity was deduced from the conventional deep-channel viscometer theory (1). The results of these measurements are shown in Fig. 4 along with the curve fit,

$$
\mu^{\mathrm{s}}(C)=-0.8+80 /(1+100 \exp (-2 C)) 10^{-4} \mathrm{sP} .
$$

It should be noted that although an exponential function was reported earlier (10), the present level of purity of the water substrate and the precision with which the water depth $( \pm 0.002 \mathrm{~cm})$ is now measured are superior to the earlier measurement $( \pm 0.02 \mathrm{~cm})$ and may account for the differences.

\section{GOVERNING EQUATIONS}

The governing equations are the axisymmetric Navier-Stokes equations, together with the continuity equation and appropriate boundary and initial conditions. We use a cylindrical polar coordinate system $(r, \theta, z)$ with associated velocity $(u, v, w)$, which are nondimensionalized using $r_{\mathrm{o}}$ as the length scale and $1 / \Omega$ as the time scale. The Reynolds number is $\operatorname{Re}=\Omega r_{\mathrm{o}}^{2} / \nu$. The boundary conditions on the solid boundaries are no-slip, i.e., $u=v=w=0$ on the stationary cylinder walls, and on the rotating floor $u=w=0$ and $v=r$ due to the rotation. We shall assume that the air/water interface is flat, and hence the contact angle at the air/water/solid contact line is $90^{\circ}$. In the physical experiment, we fix the location of the contact line with a grove machined above the interface on the cylinder walls; also, the Froude number, $\mathrm{Fr}=\Omega^{2} r_{\mathrm{o}}^{2} / g d$ for the range of Re considered is only of order $10^{-5}-10^{-2}$; hence $w=0$. This leaves the conditions for $v$ and $u$ on the interface to be specified.

We shall model the interface as Newtonian, using the Boussinesq-Scriven (8) constitutive relation, where we allow 
the surface viscosities to vary with the surfactant concentration. For a flat interface, only the tangential stress balance plays a dynamic role. The tangential stress balance in the azimuthal direction is

$$
\frac{\partial v}{\partial z}=\hat{\mu}^{\mathrm{s}}\left(\frac{\partial^{2} v}{\partial r^{2}}+\frac{1}{r} \frac{\partial v}{\partial r}-\frac{v}{r^{2}}\right)+\frac{\partial \hat{\mu}^{\mathrm{s}}}{\partial r}\left(\frac{\partial v}{\partial r}-\frac{v}{r}\right),
$$

and in the radial direction

$$
\begin{gathered}
\frac{\partial u}{\partial z}=\frac{1}{\mathrm{Ca}} \frac{\partial \hat{\sigma}}{\partial r}+\left(\hat{\mu}^{\mathrm{s}}+\hat{\kappa}^{\mathrm{s}}\right)\left(\frac{\partial^{2} u}{\partial r^{2}}+\frac{1}{r} \frac{\partial u}{\partial r}-\frac{u}{r^{2}}\right) \\
+\frac{\partial \hat{\mu}^{\mathrm{s}}}{\partial r}\left(\frac{\partial u}{\partial r}-\frac{u}{r}\right)+\frac{\partial \hat{\kappa}^{\mathrm{s}}}{\partial r}\left(\frac{\partial u}{\partial r}+\frac{u}{r}\right),
\end{gathered}
$$

where $\mathrm{Ca}=\mu \Omega r_{\mathrm{o}} / \sigma_{0}$ is the capillary number, $\hat{\mu}^{\mathrm{s}}=\mu^{\mathrm{s}} / \mu r_{\mathrm{o}}$ and $\hat{\kappa}^{\mathrm{s}}=\kappa^{\mathrm{s}} / \mu r_{\mathrm{o}}$ are the Boussinesq numbers for shear and dilatational viscosity, respectively, and $\sigma_{0}$ is the surface tension of a clean interface. Equations [3] and [4] complete the boundary conditions for $v$ and $u$.

Since the surface tension, $\sigma$, and the surface viscosities, $\mu^{\mathrm{s}}$ and $\kappa^{\mathrm{s}}$, are all functions of the surfactant concentration $C$, we need to solve an active scalar advection-diffusion equation for $C$,

$$
\frac{\partial C}{\partial t}=-\frac{1}{r} \frac{\partial}{\partial r}(r u C)+\frac{1}{\mathrm{Pe}^{\mathrm{s}}}\left(\frac{\partial^{2} C}{\partial r^{2}}+\frac{1}{r} \frac{\partial C}{\partial r}\right),
$$

where $\mathrm{Pe}^{\mathrm{s}}=\Omega r_{\mathrm{o}}^{2} / D^{\mathrm{s}}$ is the surface Peclet number and $D^{\mathrm{s}}$ is the surface diffusion of the surfactant; $D^{\mathrm{s}}$ is estimated to be of order $10^{-5} \mathrm{~cm}^{2} / \mathrm{s}$ for typical surfactants (16). Zero-flux conditions at the annular walls for $C$, i.e., $\partial C / \partial r=0$, provide conservation of $C$ on the interface.

Due to the lack of consistent measurements of $\kappa^{\mathrm{s}}$, we use the model $\kappa^{\mathrm{s}}=10 \mu^{\mathrm{s}}$. However, since $\kappa^{\mathrm{s}}$ only appears in the radial stress balance [4], we do not expect this to make any quantifiable difference to the results as $\mathrm{Ca}$ is very small $(\mathrm{Ca}$ $\in\left(3 \times 10^{-5}, 10^{-3}\right)$ for $\left.\operatorname{Re} \in(250,8500)\right)$. In fact, Eq. [4] could be replaced by $\partial u / \partial z=1 / \mathrm{Ca} \partial \hat{\sigma} / \partial r$ since $\mathrm{Ca}\left(\hat{\mu}^{\mathrm{s}}+\hat{\kappa}^{\mathrm{s}}\right)$ and $u$ at the interface are small $(10) ; \kappa^{\mathrm{s}}$ would need to be several orders of magnitude larger before any appreciable surface viscosity effects were felt in the radial stress balance.

The numerical technique to solve the governing equations is that detailed in (10). Second-order finite differences are used to discritize space, where we have used 201 grid points in the radial direction and 101 grid points in the vertical direction (the annular gap-to-depth ratio is 2). For time, we have used an explicit second-order predictor-corrector method, with time-step $\delta t$ varying between $10^{-4}$ and $10^{-6}$ (solutions have been checked so that at steady-state, they agree to six figures with solutions using halved $\delta t$ ). The time-step needs to be smaller as $C_{0}$ is increased (the equations become stiffer with increased $C_{0}$ ) and smaller for smaller Re; however, the time to reach steady state scales with $\mathrm{Re}$, so this is not a practical problem. For $\mathrm{Re}=250$, steady state is reached by $t \sim 10$, and for $\operatorname{Re}=8500$ by $t \sim 200$.

\section{RESULTS}

Measurements of surface azimuthal velocity $v^{\mathrm{s}}$, obtained via DPIV, along with computations at $\mathrm{Re}=250$ are presented in Fig. 5. The nondimensional gap coordinate is denoted by $x=\left(r-r_{\mathrm{i}}\right) /\left(r_{\mathrm{o}}-r_{\mathrm{i}}\right)$. For the two cases with relatively small concentration, $C_{0}=0.4$ and $0.8 \mathrm{mg} / \mathrm{m}^{2}$, the measurements show good agreement with the computations, verifying the Newtonian behavior of the monolayer throughout the interface. In particular for the $C_{0}=0.4 \mathrm{mg} / \mathrm{m}^{2}$ case, the measured maximum velocity exactly agrees with the computed value. The small decrease in the computed velocity profile for $C_{0}=0.8$ compared to the $0.4 \mathrm{mg} / \mathrm{m}^{2}$ case is consistent with the increase of $\mu^{\mathrm{s}}$ with $C$ (see Fig. 4). The slight difference between the measurements and the computations of the azimuthal velocity at the surface are well within the experimental uncertainty of DPIV, which is about $1-2 \%$ of the maximum velocity.

Although the Re is not very large in this case, there is secondary flow in the bulk fluid. However, the computations show that the radial velocity at the interface goes to zero asymptotically with time. Figure 6 shows how small the surface radial velocity, $u^{\mathrm{s}}$, has become (order $10^{-7} \mathrm{~mm} / \mathrm{s}$ for $C_{0}=0.4 \mathrm{mg} / \mathrm{m}^{2}$, and significantly smaller for larger $C_{0}$; it is practically in the noise level compared to $v^{\mathrm{s}}$ at order $1 \mathrm{~mm} / \mathrm{s}$ ) by time $10 / \Omega \mathrm{s}$, by which the flow is essentially at steady state. The diminished radial velocity is due to the (radial) gradients in the monolayer concentration, which oppose the bulk shear stress exerted on the monolayer. Figure 7 shows the computed distribution of the monolayer across the annulus, in terms of relative concentration $C / C_{0}$, illustrating that in the steady state, the Marangoni stress prevents the weak secondary flow in this case from producing radial flow at the interface, much like a solid no-slip wall does (10). The experiments confirm this and show that the radial velocity is only of the order of the noise in the measurements.

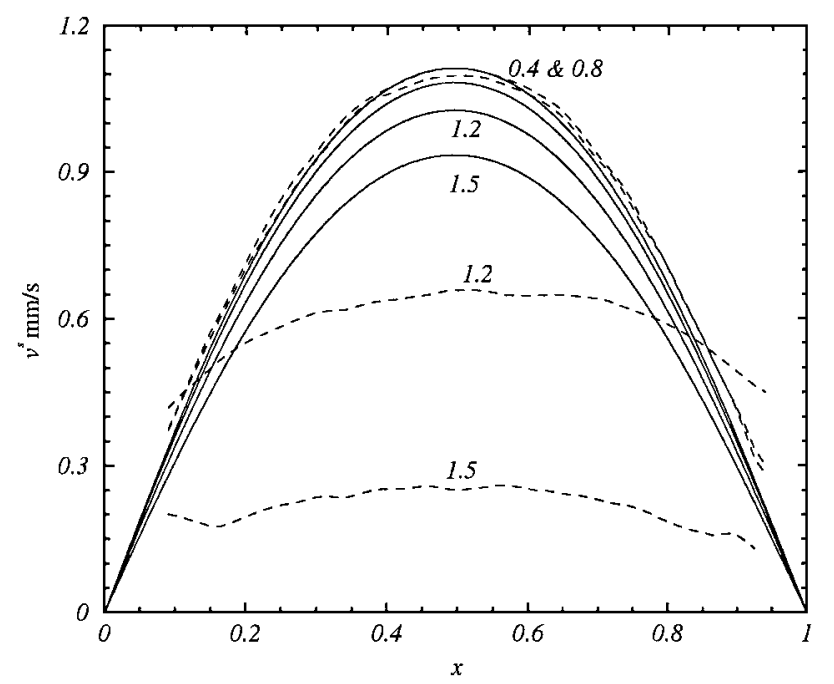

FIG. 5. Azimuthal velocity profiles at the interface driven with $\mathrm{Re}=250$ for $C_{0} \mathrm{mg} / \mathrm{m}^{2}$ as indicated; broken lines are measurements using DPIV and the solid lines are computed. 


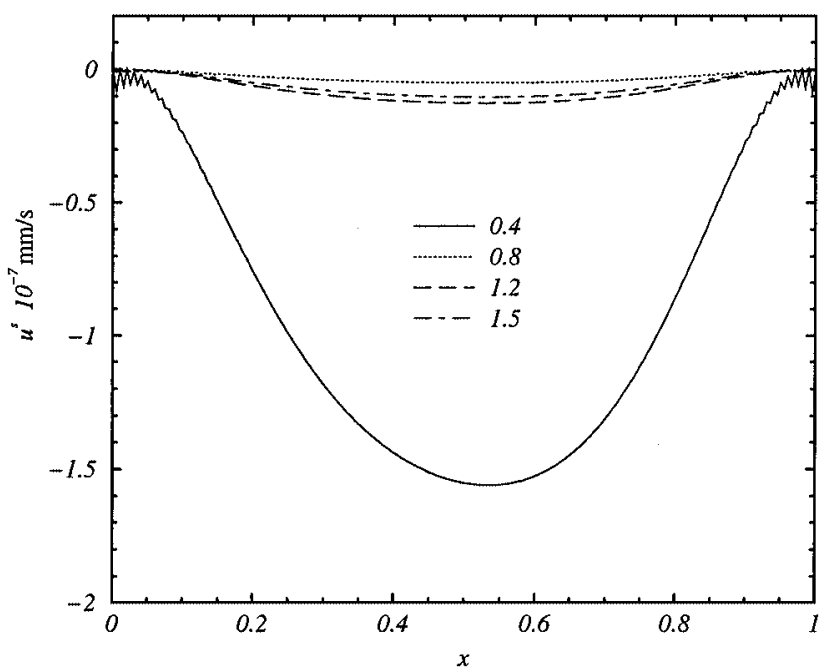

FIG. 6. Computed profiles of surface radial velocity $u^{\mathrm{s}}$ at an air/water interface with hemicyanine monolayer initially uniformally distributed with concentration $C_{0} \mathrm{mg} / \mathrm{m}^{2}$ (as indicated), driven with $\mathrm{Re}=250$.

It should be noted that the equation-of-state is nearly flat at $C=0.4 \mathrm{mg} / \mathrm{m}^{2}$; thus a comparatively larger concentration gradient is required for the $C_{0}=0.4$ case to balance the bulk shear stress than for the cases with larger $C_{0}$, including $0.8 \mathrm{mg} / \mathrm{m}^{2}$. However, the magnitude of the relative change in surface concentration is only of order $10^{-4}$ for the $C_{0}=0.4$ case and an order of magnitude smaller yet for the cases with larger $C_{0}$.

The decrease in concentration for large $x$ and increase at small $x$ is due to the overturing bulk flow in the counterclockwise direction $(5,10,19)$. It is also interesting to note that the departure from uniform distribution of the monolayer is smaller for the $C_{0}=0.8$ case than the two cases with larger $C_{0}$. This behavior is consistent with the nonlinearity in the equation-of-state, $\sigma(C)$, and the interaction between the Marangoni stress and

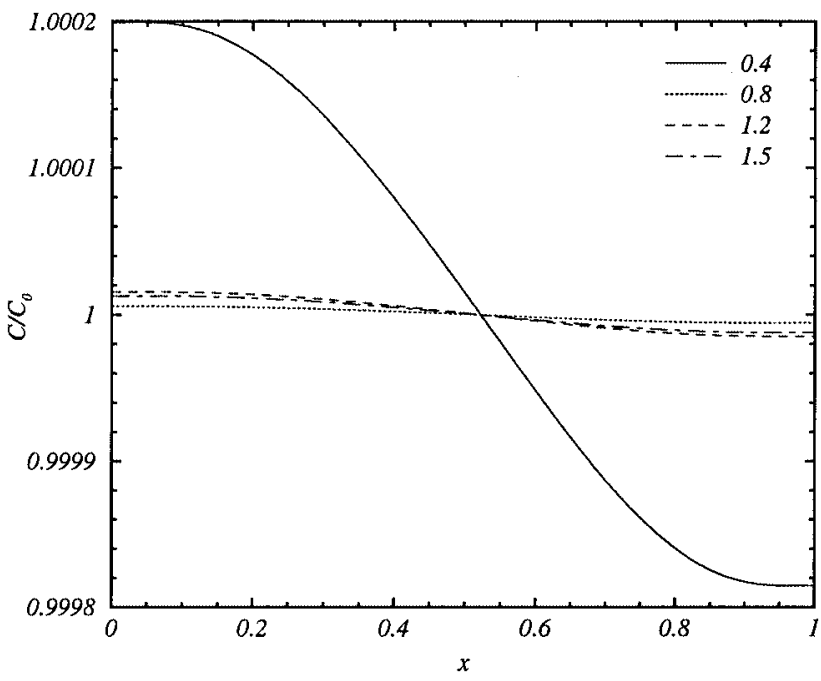

FIG. 7. Computed concentration profiles of a hemicyanine monolayer on an air/water interface driven with $\mathrm{Re}=250$.

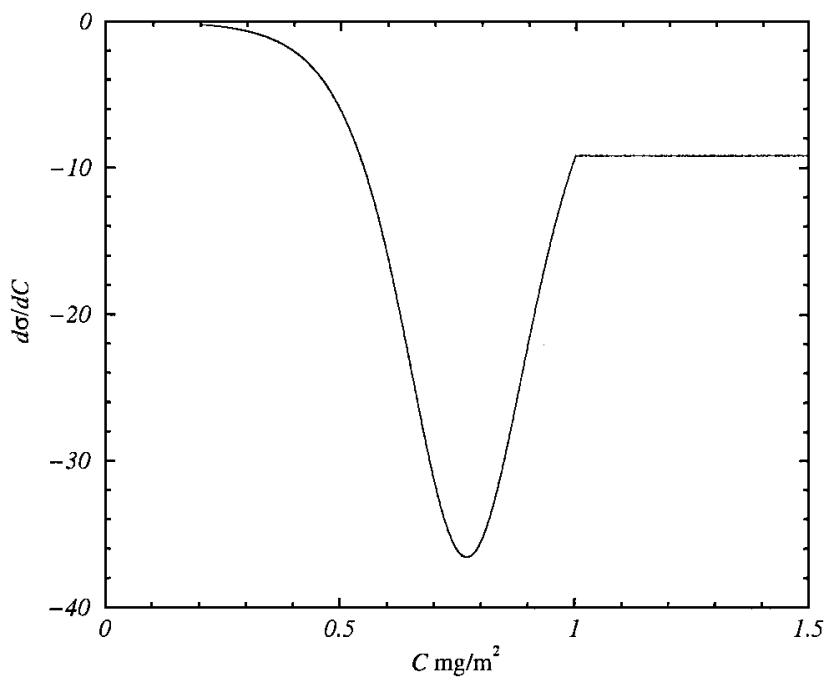

FIG. 8. Elasticity, $\partial \sigma / \partial C$, vs $C$, based on the fit [1] to hemicyanine's equation-of-state.

the bulk flow. The plot of $\partial \sigma / \partial C$, presented in Fig. 8, shows that the magnitude of the surface elasticity is at an extrema for $C \approx 0.8 \mathrm{mg} / \mathrm{m}^{2}$. Thus, near this concentration, the smallest amount of concentration gradient is needed to balance the bulk shear stress. Based on this and all the earlier results $(10,5)$, the effect of the nonlinearity of the equation-of-state can be considered well understood.

For the two cases with larger initial concentration, $C_{0}=1.2$ and $1.5 \mathrm{mg} / \mathrm{m}^{2}$, the computations show a decrease in the magnitude of velocity of about 5 and $9 \%$, respectively, from the profile for the $0.8 \mathrm{mg} / \mathrm{m}^{2}$ case (see Fig. 5). This is consistent with the nonlinear increase in $\mu^{\mathrm{s}}$ with $C$. However, the measurements at these initial concentrations show completely different behavior. Not only is the magnitude of the maximum velocity significantly less than the corresponding predictions (37 and $72 \%$ less than predicted for the 1.2 and $1.5 \mathrm{mg} / \mathrm{m}^{2}$ cases, respectively), the shape of the velocity profile is qualitatively different. The flattening of the velocity profile is indicative of shear thinning of the monolayer film (see $(11,9)$ for further examples of nonNewtonian shear thinning in other monolayers). The comparison with the computations shows that the phase transition at $C \approx$ $0.9 \mathrm{mg} / \mathrm{m}^{2}$, inferred from the equation-of-state, may demarcate Newtonian vs non-Newtonian behavior, at least at this Re.

Apart from the flattened nature of the measured velocity profiles for the $C_{0}=1.2$ and $1.5 \mathrm{mg} / \mathrm{m}^{2}$ cases, their centerline velocity values differ substantially with the computed values, as shown in Fig. 5. The reason is again due to the non-Newtonian nature of hemicyanine at those concentration levels. For both of these concentrations, the monolayer is apparently more viscous than the predictions. The stainless steel deep channel that was used to measure $\mu^{\mathrm{s}}$ is of much smaller dimensions than the optical channel used for DPIV measurements and subsequently has a greater rotation rate, $\Omega$, for a given Reynolds number. The $\mu^{\mathrm{s}}$ values are based on centerline velocity measurements 
made in the conventional deep channel. A relatively long time (about $15 \mathrm{~min}$, corresponding to 15 revolutions) was allowed for the stainless steel deep channel to operate with the spread monolayer prior to particle speed measurements (for the determination of $\mu^{\mathrm{s}}$ ). At the Reynolds number used (nominally 250), the Newtonian flow reaches steady state in about one revolution of the floor. Although, all of the measurements of $v^{\mathrm{s}}$ in Fig. 5 were also taken after 15 min from startup, that is less than 4 revolutions of the floor, but still a long time after the Newtonian flow reaches steady state. Measurements of the azimuthal velocity at the center of the conventional deep channel made as a function of time for three cases, $C_{0}=0.8,1.2$, and 1.5 are shown in Fig. 9. The long time for the surface velocity with $C_{0}=1.2$ and 1.5 to reach steady state (relative to the time for the Newtonian flow to reach steady state) is an indication of the non-Newtonian relaxation time associated with hemicyanine at those concentrations and subjected to that level of applied shear. For $C_{0}=1.2$ and 1.5 , we conclude that the interface had not yet annealed; for the lower $C_{0}$ cases, no such annealing was observed. For these concentrations, the velocity increases with time until about $1000 \mathrm{~s}$, or 15 rotations of the floor. After significant fluctuations at about this time, indicative of inhomogeneous and/or nonisotropic monolayer behavior, the monolayers reach a steady-state velocity. These steady-state velocities were used to determine $\mu^{\mathrm{s}}$ using the classical deep channel theory (13) and were shown in Fig. 4. Note that the computed $v^{\mathrm{s}}$ (Fig. 5) for all $C_{0}$ values agree with the centerline $v^{\text {s }}$ measured in the deep channel used to determine $\mu^{\mathrm{s}}$, after the interface had asymptotically reached steady state. The time-dependent change in $\mu^{\mathrm{s}}$ for the monolayers with $C_{0}=1.2$ and 1.5 is analogous to thixotropic bulk fluids for which the shear stress generated in a constant-shear-rate experiment decreases with time $(17,18)$.

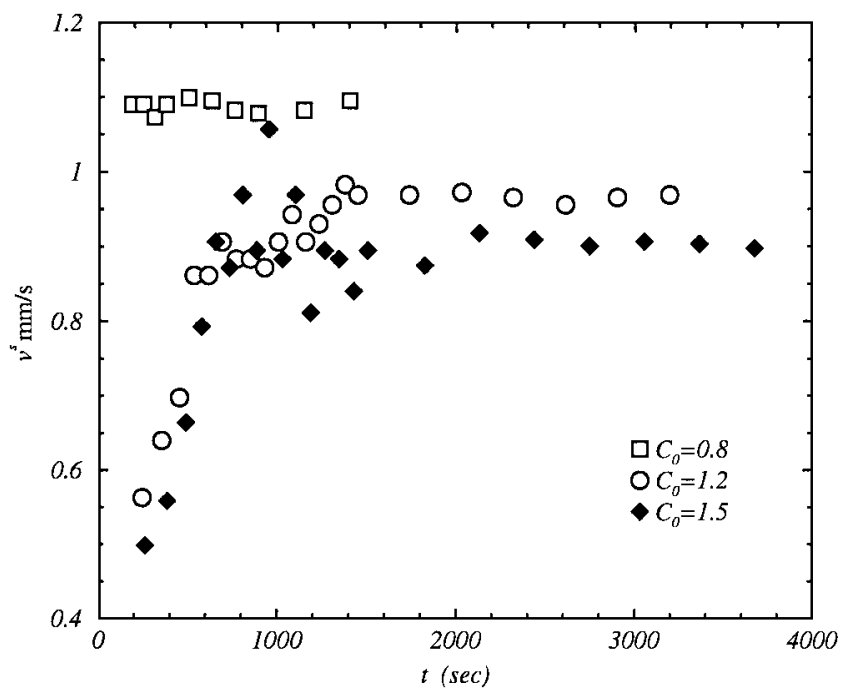

FIG. 9. Centerline azimuthal velocity measured in a conventional deepchannel viscometer vs time (in seconds) following the startup of the floor rotation, for an air/water interface with a hemicyanine monolayer of surface concentration as indicated (in $\mathrm{mg} / \mathrm{m}^{2}$ ).

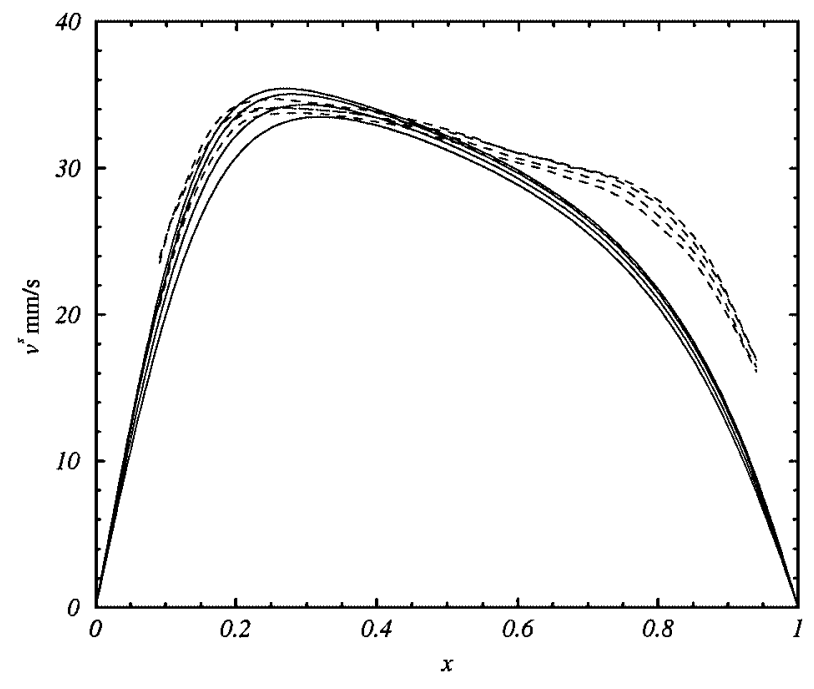

FIG. 10. Azimuthal velocity profiles at the interface driven with $\mathrm{Re}=8500$ for $C_{0}=0.4,0.8,1.2$, and $1.5 \mathrm{mg} / \mathrm{m}^{2}$; broken lines are measurements using DPIV and the solid lines are computed.

The surface azimuthal velocity profiles for a much larger Reynolds number, $\operatorname{Re}=8500$, are shown in Fig. 10. The figure shows the measured (dashed lines) and computed (solid lines) profiles across the annular gap. Although some differences exist between the measurements and the computations, the overall agreement is good for all four $C_{0}$. The peak values of $v^{\mathrm{s}}$ are in good agreement between the experiments and the computation; however, the measurements show the peak occurring at smaller $x$ for all four cases. This trend may be explained by the slight amount of depth averaging associated with the DPIV measurements, due to the finite thickness of the laser light sheet. Although the light sheet thickness is the same for all the measurements, the boundary layer at the interface is significantly thinner at $\operatorname{Re}=8500$ than at $\operatorname{Re}=250$, and the gradient $\partial v / \partial z$ is greater at larger $x$; consequently, the detrimental effect of depth-averaging is more pronounced near the outer annular wall and for larger Re.

The monolayer concentration profile for the cases shown in Fig. 10 are present in Fig. 11. Only at the smallest initial concentration, $C_{0}=0.4 \mathrm{mg} / \mathrm{m}^{2}$, is there a significant decrease (order $10 \%$ ) in the relative concentration. Although the decrease in concentration is three orders of magnitude larger at $\mathrm{Re}=8500$ than at 250 for this case $\left(C_{0}=0.4 \mathrm{mg} / \mathrm{m}^{2}\right)$, the secondary flow is not strong enough to completely clear any part of the interface. As with the lower Re case, the surface radial velocity diminishes; this was also confirmed by the measurements.

Computations were also performed at smaller $C_{0}$ in order to determine the $C_{0}$ for which the secondary flow can completely clear a portion of the interface (for $\operatorname{Re}=8500$ ). Figure 12 shows that for $C_{0}<0.3 \mathrm{mg} / \mathrm{m}^{2}$, the flow at $\mathrm{Re}=8500$ can sweep the interface free of the monolayer near the outer cylinder. The calculations show that for initial concentration of $C_{0}<0.1 \mathrm{mg} / \mathrm{m}^{2}$, the secondary flow can clear at least the outer half of the channel. 


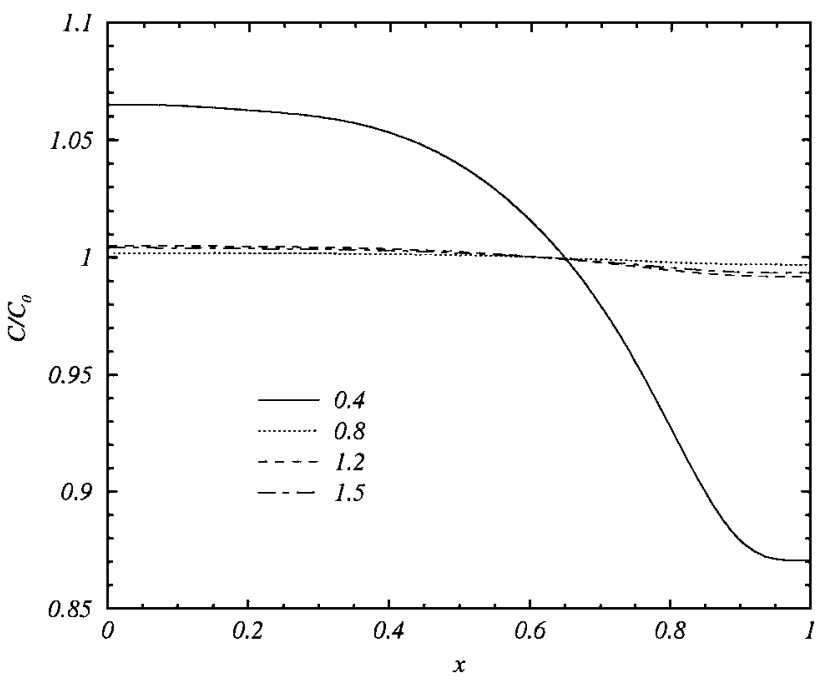

FIG. 11. Concentration profiles of a hemicyanine monolayer on an air/water interface driven with $\operatorname{Re}=8500$, for $C_{0}$ as indicated.

The results indicate that monolayers over the entire range of concentration behave Newtonian at the larger Re. This suggests that flow with sufficient inertia can "liquify" the monolayer and that the interfacial flow behavior is Newtonian. Similar shearrate behavior has been observed by (22) in a different Langmuir monolayer.

As a final note, a comparison between the present results and computations presented earlier (10) show a large difference in the initial concentration below which the interface may be partially cleaned. The results presented in (10) were based on a curve fit to the equation-of-state measured by (20). They indicated that for $C_{0} \approx 0.5 \mathrm{mg} / \mathrm{m}^{2}$ and less, the interface could be cleaned. Since the equation-of-state is very flat for $C<0.5 \mathrm{mg} / \mathrm{m}^{2}$, slight differences (of order 0.1 dynes $/ \mathrm{cm}$ ) can produce large

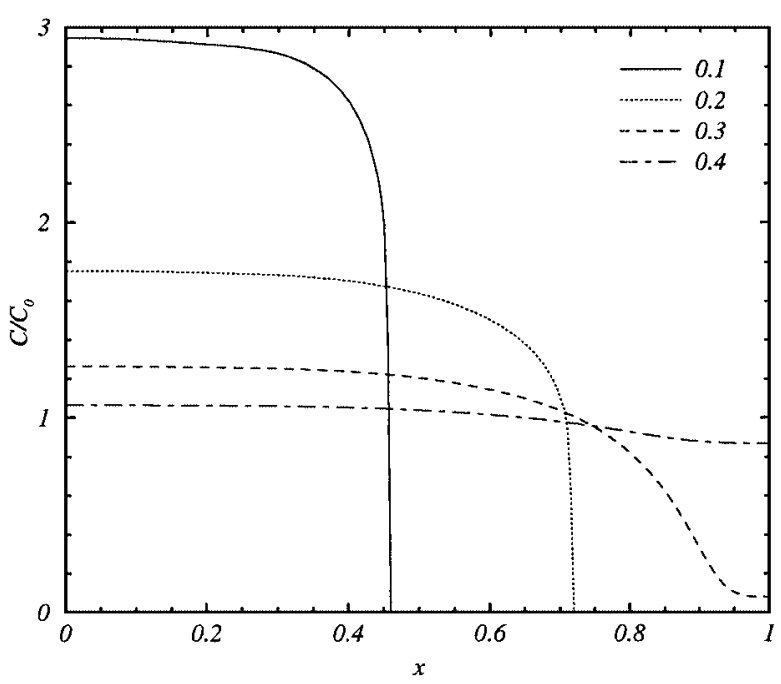

FIG. 12. Concentration profiles of a hemicyanine monolayer on an air/water interface driven with $\mathrm{Re}=8500$, for $C_{0}$ as indicated. differences in the computed results near that region. The strong dependence on the details of the nonlinear equation-of-state have also been noted elsewhere ((5) and references there in).

These results confirm the conventional wisdom that for a quantitative study, interfacial property measurements must be made for surfactants of the same purity, temperature, etc., as used in the hydrodynamic experiments to be compared with model predictions based on the measured intrinsic properties.

\section{CONCLUSION}

We have evidence of non-Newtonian behavior of a hemicyanine monolayer on an air/water interface when it is driven (sheared) with low inertia (Re in the neighborhood of Stokes flow for the bulk), when the concentration of the monolayer is greater than that at which the equation-of-state indicates of a phase transition between the liquid-expanded $\left(L_{1}\right)$ and liquid-condensed $\left(L_{2}\right)$ phases. The measured surface azimuthal velocity profiles suggest that the monolayer is experiencing shear thinning behavior in this regime. Also, apart from the thinning due to shear rate, for low Re and high $C_{0}$, we have also observed thinning of the monolayer with time at constant applied shear. This all suggests that in this regime the monolayer may be inhomogeneous and/or nonisotropic.

When the interface is driven with greater inertia from the bulk flow (i.e., larger Re), it flows like a viscous Newtonian interface with surface shear viscosity that is only a function of the thermodynamic state (i.e., $C$ and temperature), and not the flow kinematics or history. Since the high imposed shear, and associated inertia, are accounted for by solving the Navier-Stokes equations for the bulk flow, we deduce that decrepancies between the model predictions and the measured velocity profiles are indicative of non-Newtonian behavior. This is in contrast to studies that compare measurements with models derived in the limit of vanishing inertia, where secondary flows are assumed to decouple, when the interface is subjected to large shear rate; in such instances the comparison can become ambiguous.

As a final note, it would be useful to have microscopic observations of the structure of hemicyanine monolayers in the regimes indicating thinning with shear rate and time to determine if monolayer microstructure correlates with the observed thinning in hemicyanine, as is the case in other non-Newtonian Langmuir monolayers (11, 21-24).

\section{ACKNOWLEDGMENTS}

This work was supported by NSF Grants CTS-9803478 and CTS-9896259.

\section{REFERENCES}

1. Edwards, D. A., Brenner, H., and Wasan, D. T., "Interfacial Transport Processes and Rheology.” Butterworth-Heinemann, 1991.

2. Darhuber, A. A., Troian, S. M., Miller, S. M., and Wagner, S., J. Appl. Phys. 87, 7768 (2000).

3. Darhuber, A. A., Troian, S. M., and Reisner, W. W., Phys. Rev. E 64, 031603 (2001). 
4. Steen, P. H., in "Research Trends in Fluid Dynamics" (J. L. Lumley, A. Acrivos, L. G. Leal, and S. Lebovich, Eds.), American Institute of Physics, Woodbury, NY, p. 286, 1996.

5. Hirsa, A. H., Lopez, J. M., and Miraghaie, R., J. Fluid Mech. 443, 271 (2001).

6. Hirsa, A. H., Lopez, J. M., and Miraghaie, R., J. Fluid Mech. (2001). Submitted.

7. Gaines, G. L., "Insoluble Monolayers at Liquid-Gas Interfaces," Interscience, New York, 1966.

8. Scriven, L. E., Chem. Eng. Sci. 12, 98 (1960).

9. Kurnaz, M. L., and Schwartz, D. K., Phys. Rev. E 56, 3378 (1997).

10. Lopez, J. M., and Hirsa, A., J. Colloid Interface Sci. 229, 575 (2000).

11. Wei, L. Y., and Slattery, J. C., J. Colloid Interface Sci. 4, 399 (1976).

12. Pintar, A. J., Israel, A. B., and Wasan, D. T., J. Colloid Interface Sci. 37, 52 (1971).

13. Mannheimer, R. J., and Schechter, R. S., J. Colloid Interface Sci. 32, 195 (1970).
14. Hirsa, A., Korenowski, G. M., Logory, L. M., and Judd, C. D., Langmuir 13, 3813 (1997).

15. Slattery, J. C., "Interfacial Transport Phenomena," Springer-Verlag, New York, 1990.

16. Agrawal, M. L., and Neuman, R. D., J. Colloid Interface Sci. 121, 366 (1988).

17. Skelland, A. H. P., "Non-Newtonian Flow and Heat Transfer," Wiley, New York, 1967.

18. Tanner, R. I., "Engineering Rheology," Clarendon Press, Oxford, 1988.

19. Lopez, J. M., and Hirsa, A., J. Colloid Interface Sci. 206, 231 (1998).

20. Judd, C. D., "Nonlinear Optical Spectroscopy of the Air/Water Interface," Ph.D. thesis, Department of Chemistry, Rensselaer Polytechnic Institute, New York, 1996.

21. Brooks, C. F., Fuller, G. G., Frank, C. W., and Robertson, C. R., Langmuir 15, 2450 (1999).

22. Ivanova, A., Kurnaz, M. L., and Schwartz, D. K., Langmuir 15, 4622 (1999).

23. Kaganer, V. M., Möhwald, H., and Dutta, P., Rev. Mod.Phys. 71, 779 (1999).

24. Ghaskadvi, R. S., and Dennin, M., Langmuir 16, 10553 (2000). 